\title{
The Finite Element Analysis for Fork Based on ANSYS
}

\author{
Xing $\mathrm{Gu}^{1}$, Weijun Wang ${ }^{1}$, Ning Lin ${ }^{1, *}$, Gong Zhang ${ }^{1}$ and Xiang $\mathrm{Gu}^{2}$ \\ ${ }^{1}$ Guangzhou Institute of Advanced Technology, Chinese Academy of Sciences, Guangzhou, China \\ ${ }^{2}$ Anji North Automobile Inspection and Test Co., LTD, Huzhou, China \\ ${ }^{*}$ Corresponding author
}

\begin{abstract}
Introduced the important influence on the forklift overall performance and job security of the fork structure parameters. Emphasized the pros and cons of the mechanical properties of the forklift fork structure. Analyzed the simplified fork structure and the rated payload then accomplished the static analysis based on ANSYS. Acquired the maximum stress of the fork under the rated payload. Plotted the distribution curve of stress and deformation value along the fork arm. Provided a theoretical reference for the structural design of the forklift fork.
\end{abstract}

\section{Keywords-Fork; ANSYS; FEM}

\section{INTRODUCTION}

In recent years, with the rapid development of the logistics industry, forklift has got a rapid development in warehouse logistics cargo, handling stacking and short-distance transport handling tool. Forklift fork is design for supporting payloads. The forklift performance and job security quite rely on the fork strength and stiffness. Fork is rigid hanging on forklift frame and it is necessary take fork deflection vibration into consideration. So based on FEM analysis result, optimize the structure parameters of forklift fork. The analysis work base on Based on forklift RT25 from ZheJiang Noblelift Company.

\section{The Analysis Parameters}

RT25 fork groove structure made of alloy structural steel plates (35Cr) that hammered bent together. Basic structure and parameters of the fork as shown in FIGURE I.

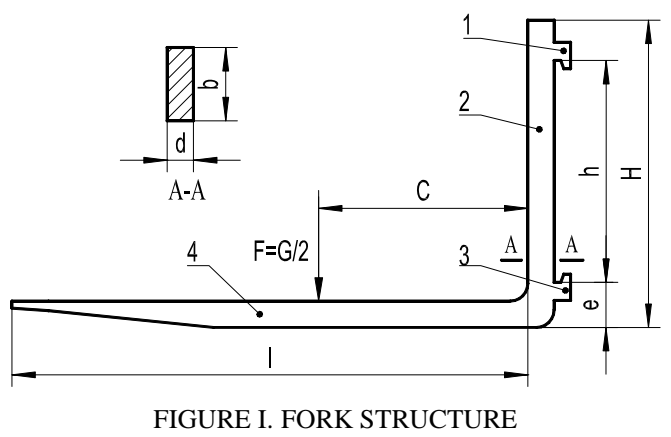

Area 4 is called fork arm and area 2 is called fork handles, 1called up hook, 3 called under hook. Length of the fork arm known as the fork length. Fork length is related with payload.
TABLE I. FORK STRUCTURE PARAMETERS

\begin{tabular}{l|l}
\hline \multicolumn{2}{l}{ RT25 forklift structure parameters } \\
\hline Fork length $\mathrm{L}$ & $1070 \mathrm{~mm}$ \\
\hline Height $\mathrm{H}$ & $650 \mathrm{~mm}$ \\
\hline Section $\mathrm{b} \times \mathrm{d}$ & $125 \mathrm{~mm} \times 45 \mathrm{~mm}$ \\
\hline Joint distance h & $477 \mathrm{~mm}$ \\
\hline From lower hook to bottom surface & $45 \mathrm{~mm}$ \\
\hline
\end{tabular}

The specification of the fork including payload $\mathrm{G}$, mass center distance $\mathrm{C}$ and the fork material and heat treatment which decide the lift capacity. The rated payload and distance C designed in RT25 typically $\mathrm{G}=2.5 \mathrm{t}$ and $\mathrm{C}=600 \mathrm{~mm}$. Other basic parameters and main structure size as shown in Figure 2.l. Fork material is $35 \mathrm{Cr}$ steel, its yield strength reached 539Mpa.

The fork top is fixed and a revolution joint is used to against the horizontal force. There is a certain gap between the Rails, load bearing hinge bearing can be reduced to activities under the circumstances (FIGURE II), fork is a statically determinate frames.

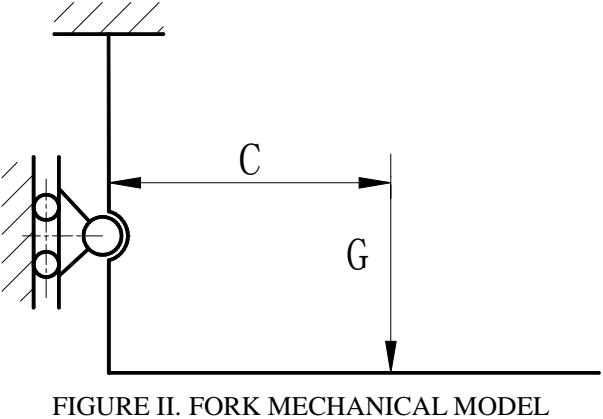

Under working condition fork under slow speed, it can be regard as at a constant speed. G/2 weight load on single fork which is 1.25 tons. In order to reduce stress concentration and stress singularity of using load instead of concentrated loads simulated bearing States of stress-strain characteristics of the fork, equivalent stress model is shown in FIGURE III

According to force load on the fork arm which are as shown in FIGURE II and FIGURE III. Based on the mechanical model got the following equations 


$$
\begin{gathered}
\int_{0}^{l}\left[p_{1}+\frac{\left(p_{2}-p_{1}\right)}{l} \cdot x\right] d x=\frac{G}{2} \times 9.8 \\
\int_{0}^{l}\left[p_{1} \cdot x+\frac{\left(p_{2}-p_{1}\right)}{l} \cdot x^{2}\right] d x=\frac{G}{2} \times 9.8 \times C \\
\text { P } 1=0.0582 \mathrm{Mpa}, \quad \mathrm{P} 2=0.125 \mathrm{Mpa}
\end{gathered}
$$

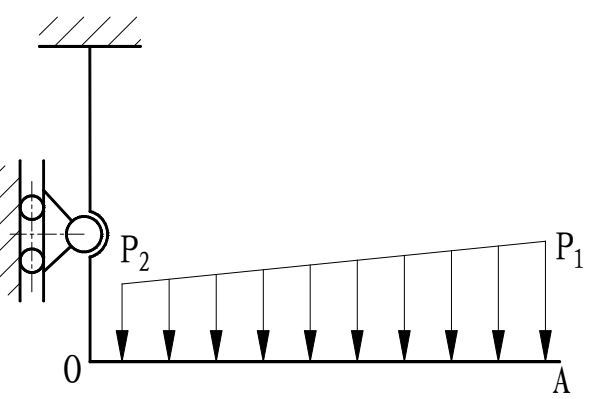

FIGURE III. LOADING MODEL

\section{FORK STATIC ANALYSIS}

Because the fork structure is relatively simple, directly modeling analysis using ANSYS preprocessor. Firstly creating a fork section, and mesh the section using the MESH200. MESH200 grid cell, the entities can be used to 1-D and 3-D, it only used as a grid reference which will not involve in the substantive analytical work after use instead of deleting (you can also use other low-dimensional unit grid, but after use to remove), very suitable for auxiliary establishment of finite element model. By using SOLID45 method to the MESH200 grid stretching generates the required solid element, the fork needed to build finite element model, as shown in FIGURE IV.

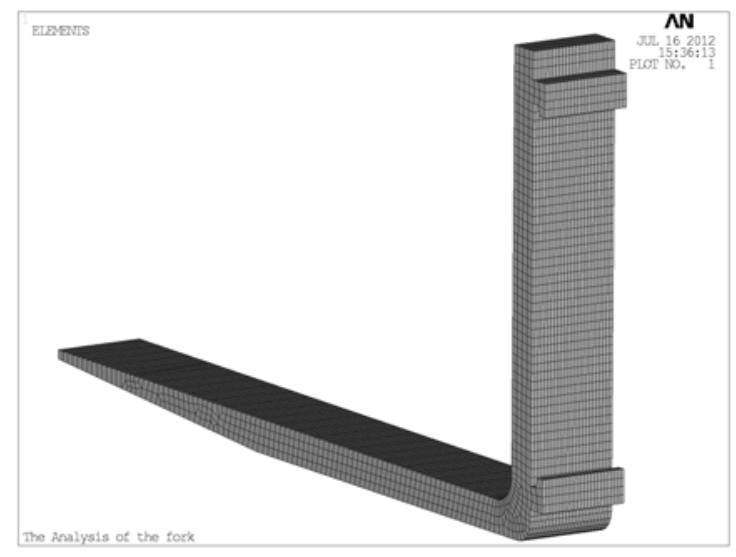

FIGURE IV. FORK FEM MODEL

According to the elastic modulus of the material set fork modal E=211Gpa, Poisson's ratio is 0.3. Consider fork separately in the bearing force characteristics in the process of Figure 2.1 illustrated a method to constraints, to hook on setting fixed constraints.

The results FIGURE V and FIGURE VI show under 2.5 ton payload, the maximum deformation occurs at the forks of fork forked arm tip, deformation of mm, Max Von Mis at the fork of the dangerous section. Stress 233Mpa factor $\mathrm{S}=2.55$.

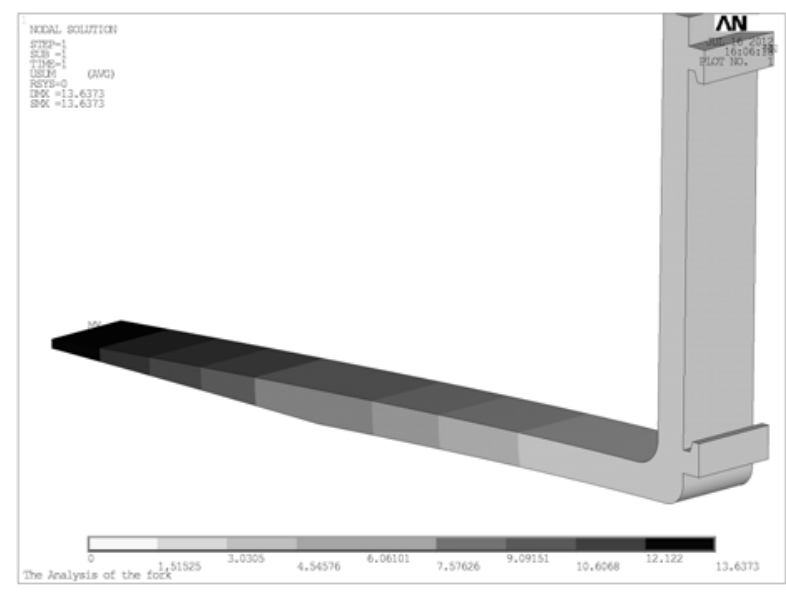

FIGURE V. FORK DEFORMATION

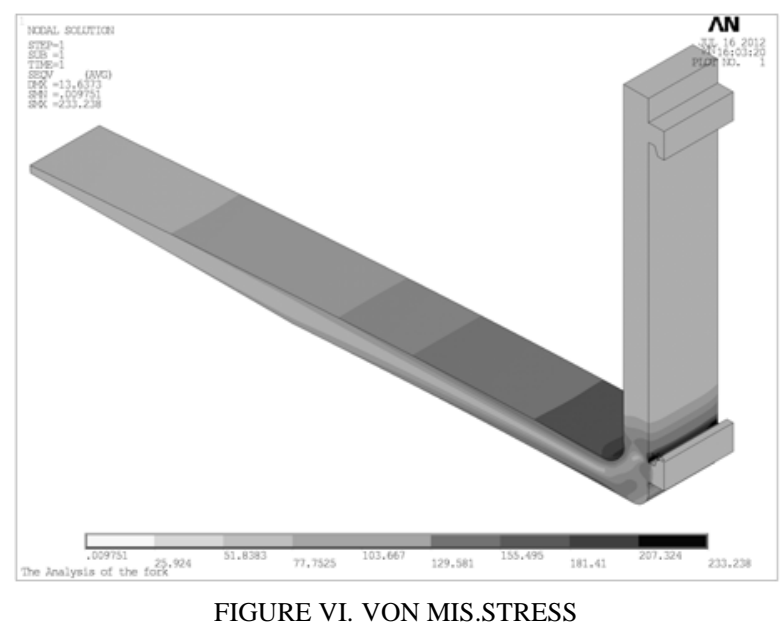

With the fork path analysis of stress and strain along the fork arm, the strain and stress can be calculated which as shown in FIGURE VII and FIGURE VIII.

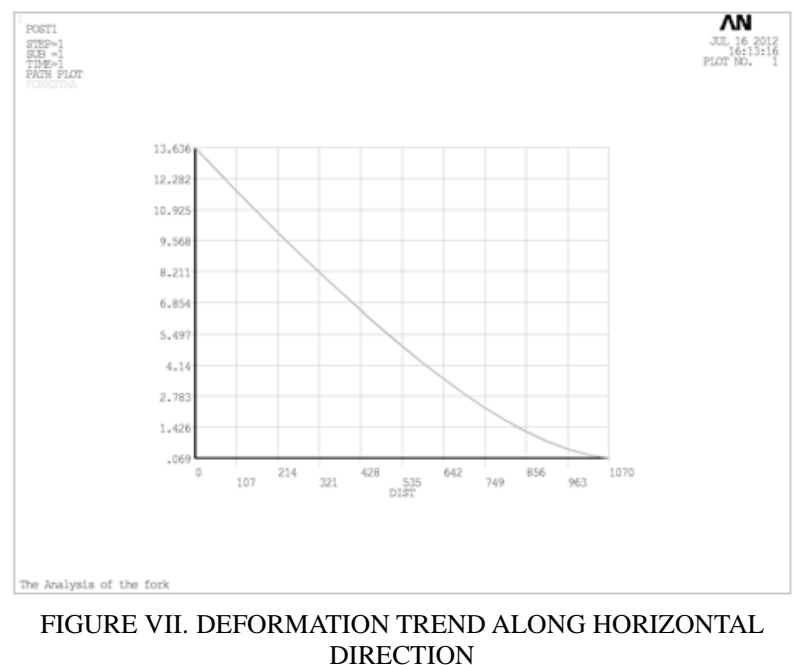

From the above analysis results, the maximum stress point is near the under hook and the maximum stress is far less than 
the permitted stress, higher reliability of structure under the load of the fork.

\section{SUMMARY}

This paper analyzes the structural stability of the fork safety importance, through the analysis of mechanical models of the fork, fork were determined in the ANSYS finite element modeling and analysis. Stress-strain characteristics were obtained through static analysis, stress-strain curves on the fork arm is drawn, for fork safety provide reference checking and optimization of the structure of the design.

\section{REFERENCES}

[1] Xing Gu , Weijun Wang, Ning Lin, Dongik Shin, The Finite Element Analysis of Batching Machine Spindle Based on ANSYS, ICMEMSCE2013, Beijing,China ,pp 216-219

[2] Kocak S, Pidaparti RMV. Three-dimensional micromechanical modeling of cord-rubber composites. J Mech Compos Mater Struct 2000;7(1):1934.

[3] Yang Daihua, Gu Xing, Lin Ning,2012, Dynamic Characteristic Analysis of the Spindle of Planer Drilling Machine Based on ANSYS, ICMDME 2012, JejuIsland, Korea,pp.699-702.

[4] J. Sim, A. Blakeborough, M. Williams, Modelling effects of passive crowds on grandstand vibration, Structures and Buildings 159 (2006) 261-272.

[5] Y. Yorozu, M. Hirano, K. Oka, and Y. Tagawa, "Electron spectroscopy studies on magneto-optical media and plastic substrate interface,” IEEE Transl. J. Magn. Japan, vol. 2, pp. 740-741, August 1987 [Digests 9th Annual Conf. Magnetics Japan, p. 301, 1982]. 